\title{
A Role for Perirhinal Cortex in Memory for Novel Object-Context Associations
}

\author{
Hilary C. Watson, ${ }^{1,2,3}$ Edward L. Wilding, ${ }^{1,2}$ and Kim S. Graham ${ }^{1,2}$ \\ ${ }^{1}$ Wales Institute of Cognitive Neuroscience and ${ }^{2}$ School of Psychology, Cardiff University, Cardiff CF10 3AT, United Kingdom, and ${ }^{3}$ Department of \\ Experimental Psychology, Oxford University, Oxford OX1 3UD, United Kingdom
}

It is debated whether functional divisions between structures in the medial temporal lobe (MTL), in particular the perirhinal cortex (PrC) and hippocampus (HC), are best conceptualized according to memory process (Diana et al., 2007; Ranganath, 2010; Wixted et al., 2010) or stimulus category (Graham et al., 2010). In the former account, $\operatorname{PrC}$ is critical for item familiarity but not recollection of associations between items and their contexts (which is instead dependent upon the HC; Ranganath et al., 2004). In the latter theory, complex object representations in $\mathrm{PrC}$ are capable of supporting memory for objects as well as for object-context associations, particularly when there is a demand to discriminate between highly visually similar objects (Cowell et al., 2010). To adjudicate between these accounts, human participants were scanned while making two different judgments about visually presented objects (is the object common or uncommon, or does the object have more edges or curves). In a subsequent, unscanned, retrieval phase, participants made item (old/new) followed by context (encoding task) judgments about previously seen and novel objects. Neural activity at encoding was separated according to the accuracy of the retrieval judgments. PrC activity predicted successful item-context judgments, a result that remained when itemmemory strength was equated across objects for which the context was remembered or forgotten. These data imply that the function of $\mathrm{PrC}$ goes beyond processing item-based memory information, contributing additionally to memory for item-context associations when the stimuli are objects (Graham et al., 2010).

\section{Introduction}

Although it is undisputed that the medial temporal lobe (MTL) is necessary for long-term memory, the contributions of different subregions-hippocampus (HC), perirhinal cortex ( $\operatorname{PrC})$, and parahippocampal cortex $(\mathrm{PhC})$-remain contentious (Diana et al., 2007; Graham et al., 2010; Wixted et al., 2010). Broadly defined, in dual-process views of MTL function, the HC is responsible for encoding and retrieving contextual information, which aligns this structure with the process of recollection. PrC, however, is necessary for familiarity, which can support judgments of prior occurrence (item memory) but does not involve recovery of contextual information (Aggleton and Brown, 1999; Diana et al., 2007; Ranganath, 2010). Evidence supporting this distinction includes findings that activity in the $\operatorname{PrC}$ at the time of encoding predicts successful item memory, while activity in the $\mathrm{HC}$ (and sometimes PhC) correlates with accurate context judgments (Cansino et al., 2002; Davachi et al., 2003; Ranganath et al., 2004; Yonelinas et al., 2005; Kensinger and Schacter, 2006).

Received Nov. 17, 2011; revised Jan. 12, 2012; accepted Feb. 3, 2012.

Author contributions: H.C.W., E.L.W., and K.S.G. designed research; H.C.W. performed research; H.C.W., E.L.W., and K.S.G. analyzed data; H.C.W., E.L.W., and K.S.G. wrote the paper.

This study was funded by the Medical Research Council (MRC; PhD studentship for H.C.W. and MRC Grant G1002149) and by the Welsh Government, which provided core funding for the Wales Institute of Cognitive Neuroscience. We thank our colleagues at the Cardiff University Brain Research Imaging Centre for help and support during data acquisition.

Correspondence should be addressed to Prof. Kim S. Graham, Wales Institute of Cognitive Neuroscience, School of Psychology, Tower Building, Park Place, Cardiff CF103AT, United Kingdom. E-mail: grahamks@cardiff.ac.uk.

DOI:10.1523/JNEUROSCI.5751-11.2012

Copyright $\odot 2012$ the authors $\quad 0270-6474 / 12 / 324473-09 \$ 15.00 / 0$
This view has been challenged by functional magnetic resonance imaging (fMRI) findings in which PrC has been implicated in memory processes beyond item memory, including encoding of object-context associations (Awipi and Davachi, 2008; Staresina and Davachi, 2008, 2010; O’Neil et al., 2009; Duarte et al., 2011; Staresina et al., 2011). For example, Staresina et al. (2011) reported that activity in the HC during memory encoding predicted subsequent memory for associations involving scenes as well as objects, while activity in the PrC did so only for objects.

There are, however, some limitations to these fMRI experiments. First, PrC activity for accurate context memory has typically been observed when the context features are elements of the item itself (e.g., imagining an item in a particular color, Staresina and Davachi, 2008). It is unclear, therefore, whether the role of $\mathrm{PrC}$ extends to contexts that do not involve item feature memory (e.g., task-related contexts). Second, to date, only one experiment has shown that context memory effects in the $\operatorname{PrC}$ remain when item-memory strength is equated across correct versus incorrect context judgments made at test (Staresina and Davachi, 2010; see Wixted, 2007, and Kirwan et al., 2008, for discussion regarding the importance of controlling for item memory in such analyses). In the absence of further demonstrations, particularly on standard contextual memory tasks, these activation patterns cannot be attributed confidently to processes supporting memory for contexts, and it remains plausible that they may reflect item memory.

These concerns were addressed by using fMRI to determine how neural activity during encoding predicted memory for objects and for object-context associations. There were two encod- 


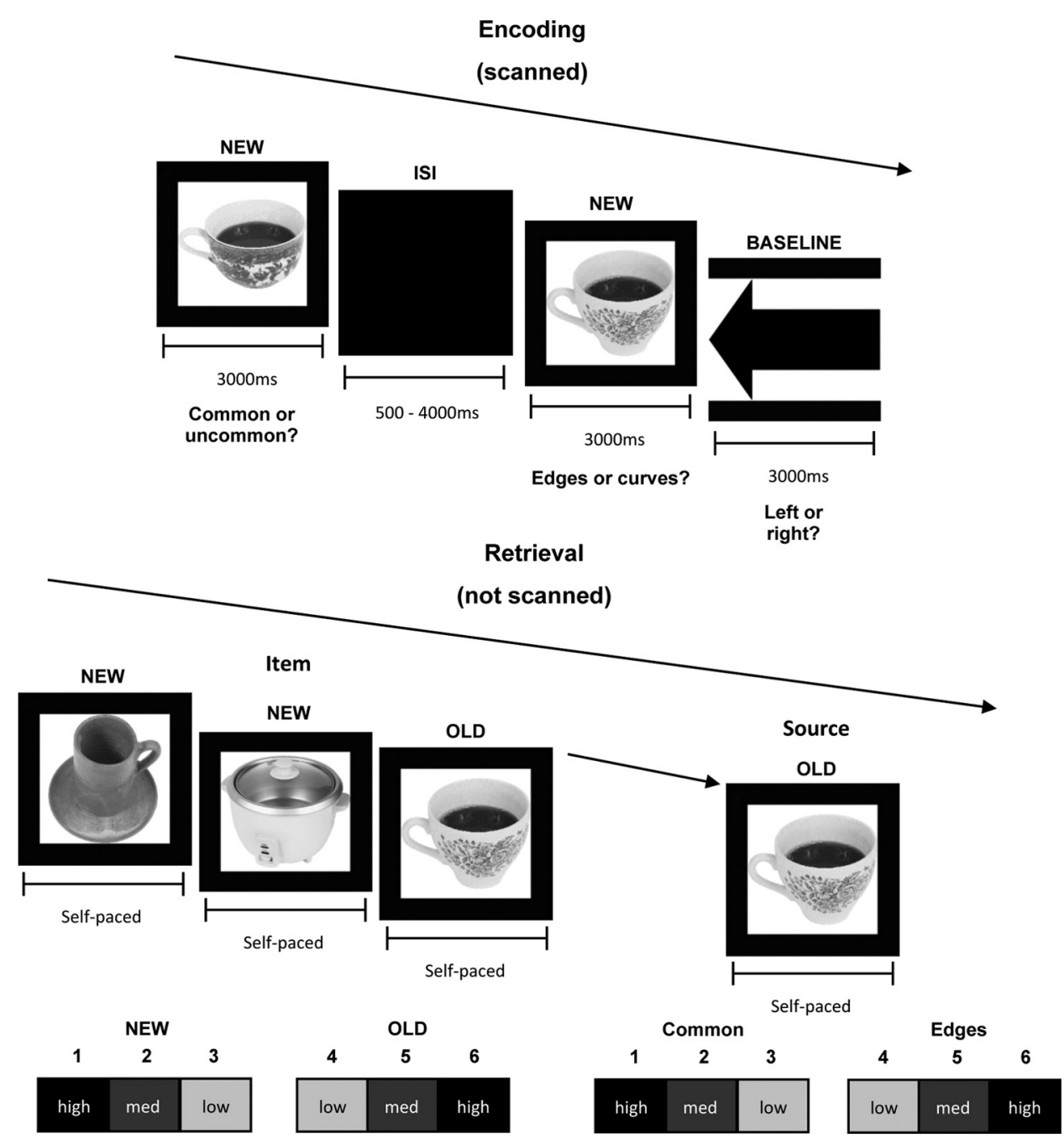

Figure 1. At encoding participants decided whether visually presented objects were common or uncommon or had more edges or curves. At retrieval participants were presented with previously studied and new objects and made old/new judgments to stimuli using a 6-point confidence scale. For objects attracting old judgments participants also indicated, again using a 6-point confidence scale, in which of the two tasks the object was originally studied. Objects were obtained from Hemera Photo 0bjects, Copyright $\odot$ 2012, Watson, Wilding and Graham and its licensors. All rights reserved.

ing tasks, in which participants made decisions about whether visually presented objects were either common or uncommon, or had more edges or curves. At retrieval, participants distinguished between studied and unstudied objects, and between the contexts in which studied objects had been encountered. Confidence judgments were obtained for both the initial object (item) and the study context decisions. Consistent with accounts in which PrC can support object context memory (Davachi, 2006; Graham et al., 2010), we predicted that $\operatorname{PrC}$ would be correlated with accurate object-context judgments, even when item-memory strength (as indexed by confidence) was taken into account.

\section{Materials and Methods}

Participants. Thirty-seven volunteers (27 female) took part. According to self-report, all were right-handed, native-English speakers with normal or corrected-to-normal vision and no neurological and/or psychiatric disorders. Three participants (two female) were excluded from the analyses due to low response accuracy (probability of a correct context judgment $<0.55$ ), and a further two (female) participants were removed due to excessive movement in the scanner. The mean age of the remaining 32 participants was 21.8 years (range, 19-30 years). All participants gave

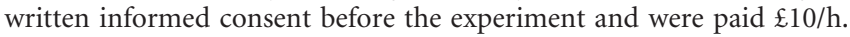

The experiment received ethical approval from the Cardiff University School of Psychology Research Ethics Committee.

Materials. The stimuli comprised 732 black-and-white images of everyday objects taken from the Hemera Photo-Objects database (Volumes I, II, and III), and two grayscale images of arrows, one pointing left, the other right, for the active-baseline task (Fig. 1). Twelve objects were used in a practice session, and of the remaining 720 objects, 360 were presented to participants in the encoding and retrieval phases, and the other 360 as new items in the retrieval phase only. The object stimuli were selected to contain an equal number of items with a high degree of semantic and visual feature overlap, and these similar items were distributed across the encoding and retrieval phases. For example, two pictures of teacups, one in each task, were presented during encoding. During retrieval, the same two teacups were presented again along with two novel teacups (Fig. 1).

Experimental tasks and procedure. Images were projected from a stimulus presentation machine to the screen within the scanner, which was manually adjusted for each participant to ensure the image was centered correctly. The MR projector system comprised a Canon SX60 LCOS projector, coupled to a Navitar SST300 zoom converter lens. To collect participant responses, a right-hand MR compatible button box was used. The MRI data were collected on a General Electric 3-T HDx MRI system using an 8 channel receiver-only head coil. 
Participants were scanned at encoding, with a surprise test phase after the scanning session (Fig. 1). There were three study runs in the encoding phase, separated by a short break just long enough to stop and start the scanning runs. Each run contained 120 object and 30 active-baseline trials. Study objects were presented again in the retrieval phase, alongside unstudied objects. The order of object and active-baseline trials at study, and object trials at test, was randomized for each participant.

Study objects were presented in the center of the screen against a black background for $3000 \mathrm{~ms}$, separated by a jittered interstimulus interval (ISI; 500-4000 ms; mean, $2250 \mathrm{~ms}$ ), during which the screen remained black. Participants had to indicate whether the study object was common or uncommon, or whether it contained more edges or curves. The prompt for the kind of judgment required appeared and remained at the bottom of the screen along with each object. Within each run, half of the objects were presented in the common/uncommon task, the remainder in the edges/curves task in a random order. The active-baseline trials were intermixed with, and were based on the same timings as, the object trials. Participants had to indicate with their right index and middle fingers whether an arrow shown at fixation pointed to the left or right.

The retrieval task (which was not scanned) also comprised three runs; participants progressed through these at their own pace taking as long a break between runs as they wished. Each run comprised 120 studied (old) and 120 unstudied (new) objects. There was no correspondence between the runs in which objects were encountered at encoding and retrieval. Objects were presented alone for $2500 \mathrm{~ms}$, after which a prompt for a self-paced item (old/new) decision appeared below the object (Fig. 1). This prompt described a six-point old/new confidence scale, with the following response keys: 1 , high-confidence new; 2 , medium-confidence new; 3, low-confidence new; 4, low-confidence old; 5, mediumconfidence old; 6 , high-confidence old. If the response was new, the experiment continued to the next trial. If the response was old, participants were prompted to make a self-paced context judgment, indicating whether the item had been processed at study in the common/uncommon or edges/curves task, again using a six-point confidence scale: 1 , high-confidence common/uncommon; 2, medium-confidence common/ uncommon; 3, low-confidence common/uncommon; 4, low-confidence edges/curves; 5 , medium-confidence edges/curves; 6 , high-confidence edges/curves. The context prompt replaced the item-memory prompt below the object and remained onscreen until a response was made. Participants were encouraged to make use of the entire scale (Yonelinas et al., 2005) for itemconfidence judgments, and for the context decision were instructed to use low-confidence response options (3, low-confidence common/uncommon; or 4, low-confidence edges/curves) when they felt they were guessing.

Participants practiced both the encoding and retrieval tasks. They saw six objects and four baseline trials in a random order before scanning. After scanning, the retrieval phase practice comprised the six objects they saw before scanning and six new objects. This was completed before the retrieval phase proper. For each response in the retrieval practice phase, participants were asked to explain the reasons for their responses, to ensure they understood the task and the use of the confidence scales. All stimulus parameters and timings in practice phases matched those described above.

A functional localizer procedure was run before the encoding phase. The design was based on previously used tasks where contrasts across conditions were used to locate stimulus-specific areas in extrastriate cortex (Peelen and Downing, 2005; Downing et al., 2006; Epstein et al., 2006; Mundy et al., 2009). Participants saw scenes, objects, and scrambled objects in separate blocks, each of $16 \mathrm{~s}$ duration. There were three groups of 12 blocks, with each group separated by a $16 \mathrm{~s}$ fixation-only block. Within each group, there was an equal number (4) of object, scene, and scrambled-object blocks. Presentation order of object, scene, and scrambled-object blocks was fully counterbalanced. Within each block, 16 pictures were presented for $300 \mathrm{~ms}$, each with a $450 \mathrm{~ms}$ ISI. To encourage attention to each picture, participants indicated whenever pictures appeared twice in succession (1-back task). Presentation of pictures within blocks was pseudo-random: immediate repeats occurred at least twice per block. Responses were made with the right index finger. There was no overlap between the pictures in the main task and the localizer task.
fMRI scanning parameters and image preprocessing. The same gradientecho echoplanar imaging (EPI) sequence was used for the subsequent memory task and the functional localizer. Forty-five slices were collected per image volume covering the whole-brain. Scanning parameters for the EPI sequence were as follows: repetition time/echo time (TR/TE) 2750 $\mathrm{ms} / 35 \mathrm{~ms}$; flip angle (FA) $90^{\circ}$; slice thickness $2.4 \mathrm{~mm}(3.4 * 3.4 * 2.4 \mathrm{~mm}$ voxel) with a $1 \mathrm{~mm}$ interslice gap; data acquisition matrix GE-EPI $644^{*} 64$; field of view (FOV) $220 * 220 \mathrm{~mm}$; and ASSET (acceleration factor). In addition, the first frames were dropped to allow for signal equilibrium. Slices were acquired with a $30^{\circ}$ oblique axial tilt relative to the anteriorposterior commissure line (posterior downward). To correct for geometrical distortions in the EPI data due to magnetic-field inhomogeneity, a map of the magnetic field was produced from two 3D spoiled gradient recalled (SPGR) images acquired during the scanning session (Jezzard and Balaban, 1995). The SPGR acquisitions were prescribed using the same slice orientation as the EPI data to be unwarped. Parameters for the SPGR acquisitions were as follows: TE, $7 \mathrm{~ms}$ and $9 \mathrm{~ms}$; TR, $20 \mathrm{~ms}$; FA, $10^{\circ}$; data acquisition matrix, $128 * 64 * 70$; FOV, $384 * 192 * 210 \mathrm{~mm}$. Anatomical images were acquired using a standard T1-weighted sequence comprising 178 axial slices (3D FSPGR). Scanning parameters were as follows: FA, $20^{\circ}$; data acquisition matrix $256^{\star} 256^{\star} 176$; FOV, $256^{\star} 256$ $\star 176 \mathrm{~mm}$, and $1 \mathrm{~mm}$ isotropic resolution.

Image preprocessing was performed using FEAT (FMRI Expert Analysis Tool) Version 5.63, which is part of the FMRIB Software Library (FSL, www.fmrib.ox.ac.uk/fsl). The following prestatistics processing was applied; motion correction using MCFLIRT (FSL, Jenkinson et al., 2002); nonbrain removal using BET (Brain Extraction Tool; FSL, Smith, 2002); spatial smoothing using a Gaussian kernel of FWHM $5 \mathrm{~mm}$; mean-based intensity normalization of the entire $4 \mathrm{D}$ dataset by the same multiplicative factor; high-pass temporal filtering (Gaussian-weighted least-squares straight line fitting, with $\sigma=50 \mathrm{~s}$ ). Phase information from the two SPGR images was unwarped using PRELUDE (FSL, Jenkinson, 2003). The unwarped phase images were then subtracted and the resulting fieldmap was used to unwarp the EPI data using FUGUE (FSL, Jenkinson, 2003). Time-series statistical analysis was performed using FILM with local autocorrelation correction (FSL, Woolrich et al., 2001). Registration to high resolution 3D anatomical T1 scans (per participant) and to a standard Montreal Neurological Institute (MNI-152) template image (for group average) was performed using FLIRT (FSL, Jenkinson and Smith, 2001; Jenkinson et al., 2002). Coordinates $(x, y, z)$ of activity are reported in MNI space.

After preprocessing the three object encoding runs for each fMRI time series for each participant, the data were submitted to a (random effects) general linear model, with one predictor that was convolved with a standard model of the hemodynamic response function (HRF) for each event-type. For the subsequent item-memory analysis, there were seven regressors formed for the encoding data separated by responses at retrieval: s6, subsequent high-confidence correct old judgment (hit); s5, subsequent medium-confidence hit; s4, subsequent low-confidence hit; s3, subsequent low-confidence incorrect new judgment (miss); s2, subsequent medium-confidence miss; s1, subsequent high-confidence miss; and active baseline (the arrow task trials). There were insufficient trial numbers to look at item-confidence effects separated according to encoding task. Moreover, as preliminary analyses of the subsequent context memory data revealed no interactions involving encoding task in any of the functional regions of interest (fROIs; all $F_{(\leq 3, \leq 93)} \leq 1.53, p>0.20$ ), the context memory data were also collapsed across task.

The formation of regressors in the context memory analysis was constrained by the distributions of correct and incorrect responses at retrieval. Five regressors were formed: (1) subsequent miss (sMM: s1, s2 and $\mathrm{s} 3$ combined); (2) subsequent hit-miss ( $\mathrm{sHM}$ - - objects identified as old for which the context judgment was incorrect; (3) subsequent lowconfidence hit-hit (sLCHH) - objects identified as old receiving a correct low-confidence correct context judgment; (4) subsequent confident hit-hit $(\mathrm{s} \mathrm{CHH})$-items identified as old receiving a correct high or medium-confidence context judgment; and (5) active baseline.

Parameter estimates relating the height of the HRF response to each regressor were calculated on a voxel-by-voxel basis, via a multiple linear regression of the response-time course, to create one $\beta$ image for each 
regressor per run, per participant. Individual runs were then concatenated for each participant in a fixed effects analysis using FEAT. The subsequent parameter estimate images were then combined in a higher-level (group) FLAME analysis (FMRIB's Local Analysis of Mixed Effects; Beckmann et al., 2003; Woolrich et al., 2004).

fROI analysis. To meet the aims of the experiment, a fROI analysis was conducted. Objectsensitive voxels within different subregions of the MTL were identified, and activation changes associated with different response outcomes were investigated within these. To create unbiased object-sensitive fROIs, group-level contrasts were performed between the object and scrambled object (object $>$ scrambled object) blocks from the orthogonal 1-back functional localizer task. Although scene stimuli were included in the functional localizer task, these were not used to locate object-sensitive voxels in the current study, because when object blocks were contrasted with scenes no voxels were identified in the hippocampus. The object $>$ scrambled-object contrast was undertaken within three anatomically defined MTL

ROIs: PrC, HC, and PhC. The PrC was defined using a probabilistic map taken from Devlin and Price (2007; available at http://joedevlin.psychol. ucl.ac.uk/perirhinal.php), which was restricted to an area that comprised a $>50 \%$ likelihood of being the $\operatorname{PrC}$ in their participants $(N=12)$. The $\mathrm{HC}$ and $\mathrm{PhC}$ were defined using the hippocampus and posterior parahippocampal gyrus probabilistic maps from the Harvard-Oxford subcortical structural atlas in FSLView, which were also restricted to a probability threshold of $>50 \%$. The resulting FEAT $t$-statistics were converted to $z$-statistics and a liberal (uncorrected) voxel threshold of $p<0.01$ was applied to the data to increase the likelihood of identifying object-sensitive voxels within each anatomical region. Data are reported for individual functional ROIs that comprised 10 or more activated voxels (Fig. 2): these were identified in the left posterior HC (peak: -20 , $-42,2, z=3.30,13$ voxels), anterior HC bilaterally (left peak: $-20,-18$, $-20, z=3.26,61$ voxels; right peak: $24,-20,-18, z=2.99,13$ voxels), left posterior $\operatorname{PrC}$ (peak: $-34,-16,-28, z=3.58,14$ voxels) and anterior $\operatorname{PrC}$ bilaterally (left peak: $-34,-8,-36, z=3.63,30$ voxels; right peak: $32,-6,-34, z=3.28,20$ voxels). The localization of these HC and $\mathrm{PrC}$ voxels was confirmed at the individual subject level using published anatomical guidelines (HC: Watson et al., 1992; PrC: Insausti et al., 1998). No object-sensitive voxels were identified in the PhC.

Using Featquery from the FSL toolkit, parameter estimate values were extracted for the six-item confidence (s1-s6) and four context-memory regressors ( $\mathrm{sCHH}, \mathrm{sLCHH}, \mathrm{sHM}$, and $\mathrm{sMM}$ ) relative to active-baseline trials within the object-sensitive MTL fROIs. Parameter estimate values from each $\mathrm{fROI}$ were then scaled by the height of the effective regressor and mean voxel intensity to convert them into percentage signal change.

Using these percentage signal-change values, omnibus ANOVAs were conducted to investigate (1) activity predicting item memory [factors of fROI ( $3 \mathrm{HC}$ and $3 \mathrm{PrC}$ regions) and response category (six levels- high, medium, and low-confidence hit and miss)] and (b) activity predicting object-context memory [factors of fROI (as above) and category ( $\mathrm{sCHH}$, sLCHH, sHM, and sMM)]. Including sMM here allowed for the potential to identify item-memory effects in this contrast. For both ANOVAs, interactions between region and category were followed up by ANOVAs within each fROI. In all factorial ANOVAs, the Greenhouse-Geisser (Greenhouse and Geisser, 1959) correction for nonsphericity was applied where necessary and was indicated by adjusted degrees of freedom.

Activity predicting memory for object-context associations was also analyzed after equating for item-memory strength (see Introduction; Kirwan et al., 2008; 2010). Before controlling for strength, the itemconfidence judgments were higher, on average, for objects that attracted correct rather than incorrect context judgments (see Table 3 for details).
Hence, changes in activation between $\mathrm{sCHH}$ and sHM could reflect differences in item-memory strength rather than activity supporting object-context judgments. Following Kirwan et al. (2008), we avoided this confound by making an additional contrast between $\mathrm{sCHH}$ and $\mathrm{sHM}$ response categories that were restricted to objects that attracted a highconfidence correct item-memory judgment.

Whole-brain analysis. To examine the relationship between encoding activity and memory for objects in subregions of the MTL that may be overlooked using an fROI approach, a group level contrast was performed between the combined regressors for forgotten objects and the regressors for all objects identified correctly as old, regardless of confidence and context judgment accuracy (Davachi et al., 2003). For this hit/miss contrast, Feat group (Gaussianized) $t$-statistics were converted to $z$-statistics and thresholded using a voxel significance level of $p<$ 0.001 , uncorrected for multiple comparisons. At this probability threshold, contiguous clusters of at least 9 voxels are equivalent to a mapwise false-positive rate for the MTL (encompassing the hippocampus, parahippocampal gyrus, and perirhinal cortex) of $p<0.05$ (estimated using the Monte Carlo procedure implemented in the AlphaSim program in AFNI). Where the whole-brain analysis revealed significant patterns of activity within the MTL, the patterns were localized to the perirhinal cortex, hippocampus, or parahippocampal cortex using the probabilistic masks from the fROI analyses, in addition to published anatomical guidelines (Watson et al., 1992; Insausti et al., 1998).

\section{Results}

\section{Behavior}

For ease of exposition, the common/uncommon task will be referred to as the common task and the edges/curves task as the edges task. Old/new discrimination, collapsed across confidence and measured by $p$ (hit)- $p$ (false alarm), was above chance for objects from both tasks (common: $0.55, \mathrm{SE}=0.03, t_{(31)}=21.98$, $p<0.01$; edges: $\left.0.42, \mathrm{SE}=0.03 ; t_{(31)}=15.90, p<0.01\right)$, and superior for the common task $\left(t_{(31)}=11.84, p<0.01\right)$. The proportion of item-memory responses to old items, separated by encoding task (Table 1 ), was entered into a $2{ }^{*} 6$ ANOVA with factors of task and confidence, which revealed an interaction $\left(F_{(3.31,102.55)}=39.73, p<0.01\right)$. Bonferroni corrected paired comparisons across tasks for each of the six judgments (revised $p$ value $=0.008$ ) revealed that false alarms were more likely for objects encountered in the edges task at all levels of confidence, while correct old judgments were more likely for objects from the 
Table 1. Mean proportions (and SE) of old/new judgments to old and new objects, separated by confidence and encoding task

\begin{tabular}{lllllll}
\hline \multirow{5}{*}{ Proportion } & \multicolumn{6}{l}{ Recognition judgments } \\
\cline { 2 - 7 } & High new & Med new & Low new & Low old & Med old & High old \\
\hline Com old & $0.08(0.02)$ & $0.10(0.01)$ & $0.11(0.01)$ & $0.14(0.01)$ & $0.22(0.02)$ & $0.35(0.03)$ \\
Edges old & $0.12(0.02)$ & $0.14(0.01)$ & $0.15(0.01)$ & $0.16(0.01)$ & $0.18(0.02)$ & $0.25(0.03)$ \\
New & $0.36(0.04)$ & $0.29(0.02)$ & $0.19(0.02)$ & $0.08(0.01)$ & $0.05(0.01)$ & $0.03(0.01)$ \\
\hline
\end{tabular}

Number of common old items, 80; edges old, 180; new items, 360; Com, Common; Med, Medium.

Table 2. Mean proportions (and SE) of context judgments to objects that attracted a correct old judgment, separated by confidence and encoding task

\begin{tabular}{|c|c|c|c|c|c|c|}
\hline \multirow[b]{2}{*}{ Proportion } & \multicolumn{6}{|c|}{ Context judgment } \\
\hline & $\begin{array}{l}\text { High } \\
\text { common }\end{array}$ & $\begin{array}{l}\text { Medium } \\
\text { common }\end{array}$ & $\begin{array}{l}\text { Low } \\
\text { common }\end{array}$ & $\begin{array}{l}\text { Low } \\
\text { edges }\end{array}$ & $\begin{array}{l}\text { Medium } \\
\text { edges }\end{array}$ & $\begin{array}{l}\text { High } \\
\text { edges }\end{array}$ \\
\hline & & $0.25(0.02)$ & $0.16(0.02)$ & $0.13(0.01)$ & .01) & \\
\hline dges old & $0.04(0.01)$ & $0.07(0.01)$ & $0.09(0.01)$ & $0.22(0.02)$ & $0.29(0.02)$ & $0.28(0.03)$ \\
\hline
\end{tabular}

The average number of trials for common- and edges-old items was 130 and 106, respectively; Com, Common.

common task (the accuracy difference for low-confidence correct judgments only approached significance: $t_{(31)}=2.7, p=0.01$ ).

The likelihood of a correct context judgment was above chance for both encoding tasks (common: $0.73, \mathrm{SE}=0.02, t_{(31)}=$ 13.94, $p<0.01$; edges: $\left.0.79, \mathrm{SE}=0.02, t_{(31)}=15.05, p<0.01\right)$, and was higher for the edges task $\left(t_{(31)}=2.44, p<0.05\right.$; Table 2$)$. Context judgments for false alarms indicated a trend $\left(t_{(31)}=1.96\right.$, $p=0.06$ ) toward a bias to respond edges (probability of an edges response $=0.54, \mathrm{SE}=0.02$, collapsed across context judgment confidence). Bonferroni corrected paired comparisons (corrected $p$ value $=0.008$ ) across tasks for correct and incorrect responses at each level of confidence revealed only that the likelihood of a correct low-confidence context judgment was higher for objects encountered in the edges rather than in the common task $\left(t_{(31)}=4.26, p<0.008\right)$.

\section{Functional region of interest analysis}

We first analyzed activation changes for studied objects according to subsequent item recognition confidence within the object-sensitive MTL fROIs. The ANOVA revealed a significant $\mathrm{fROI}^{*}$ response category interaction $\left(F_{(9.10,282.10)}=2.59, p<\right.$ $0.01)$. Further analysis indicated a significant effect in the left anterior PrC fROI only $\left(F_{(3.77,116.85)}=5.53, p<0.001\right.$; for all other fROIs $F_{(5,155)}<0.98$; Fig. $\left.3 b\right)$. Bonferroni corrected pairwise comparisons ( 15 comparisons, revised $p$ value $=0.003$ ) revealed reliable or marginal differences between high-confidence hit (s6) and all other response categories ( $\mathrm{s} 6>\mathrm{s1}: t_{(31)}=4.59, p<$ $0.003 ; \mathrm{s} 6>\mathrm{s} 2: t_{(31)}=3.70, p<0.003 ; \mathrm{s} 6>\mathrm{s} 3: t_{(31)}=4.50, p<0.003$; s6 > s5: $t_{(31)}=4.69, p<0.003$; s6 $>$ s4: $\left.t_{(31)}=3.00, p=0.005\right)$. There were also differences between medium-confidence hit (s5) and low (s3) and high-confidence (s1) misses that approached significance $\left(\mathrm{s} 5>\mathrm{s} 3: t_{(31)}=2.35, p=0.03 ; \mathrm{s} 5>\mathrm{s} 1: t_{(31)}=\right.$ 2.58, $p=0.02)$.

The context memory analysis also revealed on interaction between response category and fROI $\left(F_{(6.52,201.96)}=2.05, p<0.05\right)$. Follow up one-way ANOVAs revealed reliable effects in left anterior $\left(F_{(2.09,64.88)}=9.42, p<0.001\right.$; Fig. $\left.3 c\right)$ and left posterior $\operatorname{PrC}\left(F_{(2.40,74.47)}=3.92, p<0.05\right)$. For the anterior PrC, Bonferroni corrected pairwise comparisons (six comparisons, revised $p$ value $=0.008$ ) revealed reliable differences between highconfidence correct context judgments and all other categories $\left(\mathrm{sCHH}>\mathrm{sLCHH}: t_{(31)}=4.99, p<0.008 ; \mathrm{sCHH}>\mathrm{sHM}: t_{(31)}=\right.$ 3.72, $p<0.008$; $\mathrm{sCHH}>$ sMM: $\left.t_{(31)}=7.33, p<0.008\right)$. In the posterior $\mathrm{PrC}$ fROI, there was a significant difference between
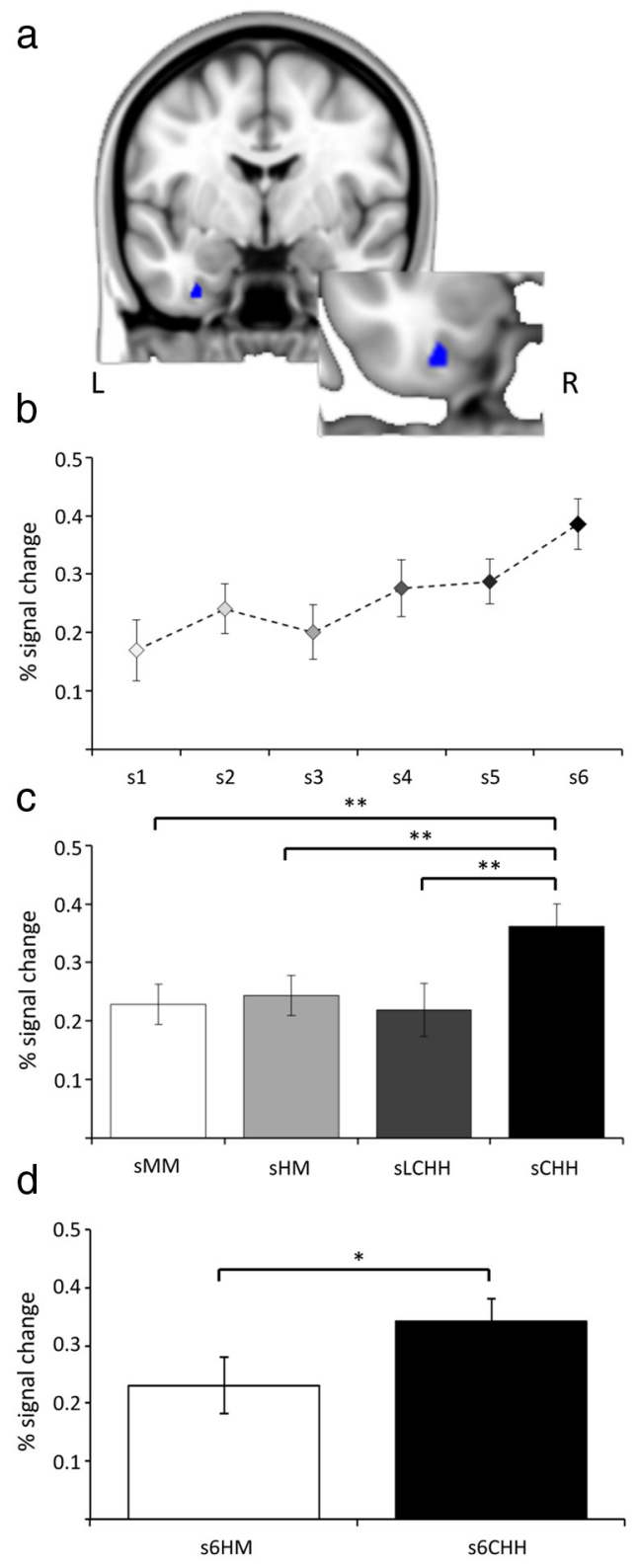

Figure 3. $\quad \boldsymbol{a}$, Coronal section and magnified image of the left anterior $\operatorname{PrC} f$ ROI. Patterns of activity at encoding from the left anterior fROl separated by $(\boldsymbol{b})$ old/new recognition memory confidence, (c) the accuracy of context judgments, and (d) the accuracy of context judgments for objects given highly confident correct old judgments. s6HM, incorrect context judgment for objects given a high-confidence correct item-memory decision; $\mathrm{s} 6 \mathrm{CHH}$, confident correct context judgment for items given a high-confidence correct item-memory decision. ${ }^{*} p<0.05 ;{ }^{* *} p<0.01$.

high-confidence correct context judgments and misses, and a marginal difference between high- and low-confidence correct context judgments $\left(\mathrm{sCHH}>\mathrm{sMM}: t_{(31)}=2.99, p<0.008\right.$; $\left.s \mathrm{CHH}>\mathrm{sLCHH}: t_{(31)}=2.55, p=0.02\right)$.

As planned, we also conducted context memory analyses that were matched for item-memory strength, comparing the degree of activation seen for $\mathrm{sCHH}$ and $\mathrm{sHM}$ responses that attracted high-confidence correct item judgments (for the proportions of confidence judgments separated by context decision, see Table 3). Pairwise comparisons between these two categories revealed a significant difference in the left PRC only $\left(t_{(31)}=2.52, p<0.02\right.$, Fig. $3 d$; and for all other fROIs, $\left.t_{(31)} \leq 1.43\right)$. 
Table 3. The proportions (and SE) of high-, medium-, and low-confidence correct old judgments made for objects that attracted high-confidence correct context judgments, medium- or low-confidence correct context judgments, or incorrect context judgments

\begin{tabular}{llll}
\hline \multirow{4}{*}{ Proportion } & \multicolumn{3}{l}{ Confidence judgments } \\
\cline { 2 - 4 } & SCHH & sLCHH & SHM \\
\hline s6 & $0.58(0.04)$ & $0.22(0.02)$ & $0.32(0.03)$ \\
s5 & $0.30(0.03)$ & $0.28(0.02)$ & $0.35(0.03)$ \\
s4 & $0.12(0.02)$ & $0.50(0.02)$ & $0.34(0.03)$
\end{tabular}

s6, subsequent high-confidence correct old judgment (hit); 55 , subsequent medium-confidence hit; $s 4$, subsequent low-confidence hit; sCHH, subsequent confident hit-hit; sLCHH, subsequent low-confidence hit-hit; sHM, subsequent hit-miss. The average number of high-confidence hits (s6) collapsed across encoding task was 108.

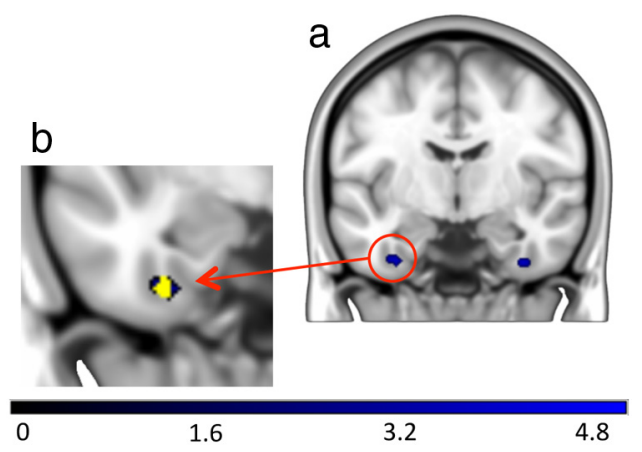

Figure 4. a, Patterns of activation revealed in the whole-brain analysis contrasts between hits and misses. In the MTL these were located in the left PrC and the right PrC/fusiform gyrus. $\boldsymbol{b}$, A magnified view of the left $\operatorname{PrC}$ cluster (blue) with the left anterior $\operatorname{PrC}$ fROI overlaid (yellow).

\section{Whole-brain analysis}

The single hit versus miss contrast revealed two significant clusters within the MTL (Fig. 4a). These were located in the left anterior $\operatorname{PrC}$ (peak: $-32,-8,-38, z=3.90,18$ voxels) and in an area bordering the right $\operatorname{PrC}$ and fusiform gyrus (peak: $36,-8$, $-38, z=3.82$, 18 voxels). There was considerable overlap (72\%) between the left anterior PrC voxels reported in the fROI analysis and the hit versus miss contrast (at peak voxel in the left anterior PrC fROI: $-34,-8,-36, z=3.30$, Fig. $4 b$ ). Furthermore, outcomes from the analysis of item and context memory effects derived from the left anterior $\operatorname{PrC}$ hit-versus-miss cluster were statistically equivalent to those obtained via the fROI analyses, including those when obtained item-memory strength was matched.

An additional whole-brain level analysis was conducted between the regressors for $\mathrm{s} \mathrm{CHH}$ and sHM items. No effects were observed in the MTL with probability set at $p<0.001$, extent at 9 voxels. When the uncorrected voxelwise probability was dropped to $p<0.01$, however, there was bilateral activation in the anterior HC (left HC peak: $-26,-22,-14, z=2.89,26$ voxels; right HC peak: $18,-10,-18, z=3.27,25$ voxels, Fig. $5 a$ ). The cluster extent threshold for an uncorrected voxel significance level of $p<$ 0.01 is 38 voxels (estimated using the Monte Carlo procedure implemented in the AlphaSim program in AFNI).

As with the fROI analysis, we investigated whether differences in activation between $\mathrm{SCHH}$ and sHM remained when the level of memory strength was equated across these two response categories. The relevant percentage signal-change values extracted from the left and right $\mathrm{HC}$ clusters for these balanced response categories were not reliably different (both $t_{(31)} \leq 1.15$; Fig. $5 b$ ).

\section{Discussion}

To determine how MTL regions support novel object-context associations, encoding-related activity for objects was investi-
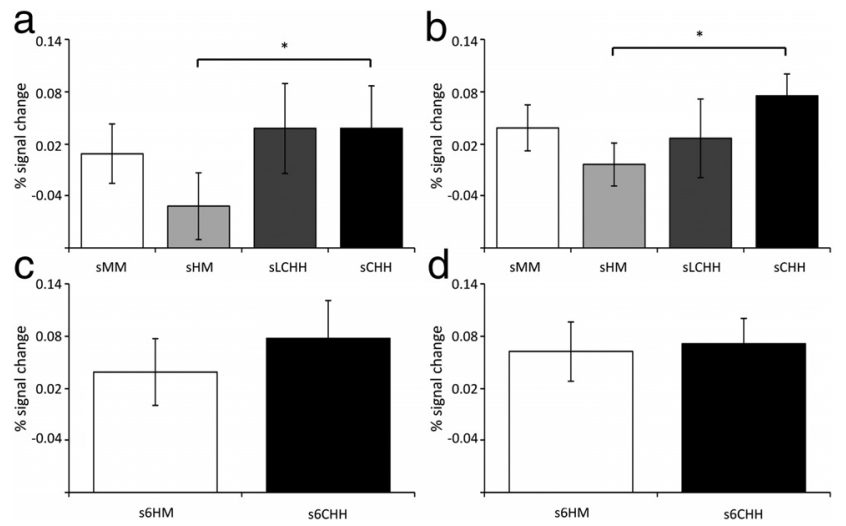

Figure 5. Top, neural activity in the left ( $\boldsymbol{a})$ and right $(\boldsymbol{b})$ anterior $\mathrm{HC}$ for forgotten objects and objects attracting high-confidence correct context judgments, low-confidence correct context judgments, and incorrect context judgments. Bottom, neural activity in the left (c) and right (d) anterior $\mathrm{HC}$ for objects attracting correct context judgments and high-confidence correct old judgments. Abbreviations are as defined in Materials and Methods, fMRI scanning parameters and image preprocessing, and in the legend to Figure $3 .{ }^{*} p<0.01$.

gated in object-sensitive voxels in the anterior PrC and $\mathrm{HC}$ (bilaterally), and left posterior PrC and HC. Left anterior PrC, and to a lesser extent left posterior PrC, predicted the accuracy of context judgments. In addition, in the anterior PrC, high-confidence correct old judgments were associated with significantly greater activity than lower confidence correct old judgments, as well as incorrect new judgments to studied objects (for all levels of confidence). Critically, the index of memory for context in anterior PrC remained even when the analysis was restricted to a contrast between objects attracting high-confidence item judgments and then either correct or incorrect context judgments.

In previous fMRI experiments, the contribution of a given MTL region to familiarity has been inferred by assuming that the neural signature of familiarity-based recognition is characterized by a roughly linear modulation in activity across varying levels of recognition confidence for old as well as new items (Ranganath et al., 2004; Daselaar et al., 2006; Haskins et al., 2008). The analyses of the data according to item confidence in this experiment did not reveal this pattern. Activity did not vary for misses at any level of confidence, nor for low- and medium-confidence correct responses. Highly confident correct old responses, however, were associated with reliably greater activity than all other response categories. While this outcome does not conform to the linear pattern that would align $\operatorname{PrC}$ with the process of familiarity, it is possible to maintain this link by appealing to statistical power and noting that (at least for $s 4-s 6$ ) there is a semblance of a linear change with increasing confidence (Fig. $3 b$ ).

Critically, however, when anterior PrC activity during encoding was separated according to context memory accuracy, activity for forgotten objects, objects assigned to the incorrect context, and objects attracting low-confidence correct context judgments did not differ, but their activity levels were lower than those associated with medium- and high-confidence correct context judgments. This nonlinear pattern has been previously aligned with recollection (Davachi et al., 2003; Yonelinas et al., 2005; Daselaar et al., 2006; Diana et al., 2010); thus, these results suggest that $\mathrm{PrC}$ can support this process.

Although there are previous examples of PrC contributions to accurate associative memory (Staresina and Davachi, 2006, 2008, 2010; Awipi and Davachi, 2008; Diana et al., 2010; Staresina et al., 2011), including a study showing that this pattern remains when 
item-memory strength is controlled (Staresina and Davachi, 2010), our finding is novel for two reasons. First, it is the first demonstration that PrC activity predicts memory for objectcontext associations when item strength is matched across correct and incorrect task-related context decisions, as opposed to judgments about an associated object context. Second, the use of a fine-grained confidence scale allowed us to demonstrate that this effect is driven by high, compared with low, confidence source decisions (s6).

In one neuroanatomical dual-process model, $\mathrm{PrC}$ and $\mathrm{HC}$ support the processes of familiarity and recollection, respectively (Aggleton and Brown, 1999; Brown et al., 2010). In recent variants of this view, however, it has been proposed that associative memory can be supported by $\operatorname{PrC}$ when an item and associated details are bound into a single memory representation (Yonelinas, 1999; Mayes et al., 2007; Montaldi and Mayes, 2010), a process termed "unitization." For example, associative memory deficits in patients with focal HC damage can be ameliorated when word pairs are encoded as a single item (Giovanello et al., 2006; Quamme et al., 2007) and $\operatorname{PrC}$ activity is greater during memory encoding for unitized than for nonunitized word pairs (Haskins et al., 2008). In a related branch of dual-process accounts, the $\mathrm{HC}$ and $\mathrm{PrC}$ are predominantly specialized for processing contextual versus item-based mnemonic information, respectively (Davachi, 2006; Diana et al., 2007; Ranganath, 2010). In these, the $\operatorname{PrC}$ can support familiarity and recollection of associated information when it comprises, or is encoded as, part of an item. These different views assume that $\operatorname{PrC}$ cannot support memory for between-domain (or item and context) associations, which "converge mainly, if not solely" within the HC (Mayes et al., 2007, p. 127, although see Park and Rugg, 2011).

Our findings, therefore, are inconsistent with these accounts, unless one believes that our encoding tasks also encouraged processing of the context as an item feature and/or that two-choice context judgments, like those used in the current experiment, might not solely isolate the recovery of contextual information (Montaldi and Mayes, 2010). We feel justified in our claim that the current data reflect recollection for object-context associations, in part because (1) our task is similar to many paradigms reported in literature, including those used in support of a HC contribution to context memory (Davachi et al., 2003; Ranganath et al., 2004) and (2) our consideration of what encompasses memory for an item versus a context was based on Ranganath's (2010) recent distinctions between items and contexts in "practice and principle" (p. 1278). An important next step, however, would be to replicate our findings using alternative tasks, such as those in which multiple contexts or item-specific contexts are used, and where alternative means of assessing memory, such as variants of remember/know paradigms (Montaldi and Mayes, 2010), are used. If we found comparable $\operatorname{PrC}$ activation for objects in a memory study in which multiple contexts were tested, it would be hard to argue that participants rely more on familiarity when only a small number of contexts are used. Furthermore, as the use of two memory contexts is the most frequently used design to investigate context memory, this concern goes beyond this study to those in which HC activation has also been elicited. Clearly, there is a requirement for memory researchers to consider novel approaches to disentangling context from item memory if we are to understand how recollection and familiarity contribute to memory judgments.

How can the current findings be explained? A growing body of neuropsychological and fMRI research has implicated the $\operatorname{PrC}$ and $\mathrm{HC}$ in perception and memory for objects/faces and scenes, respectively (Meunier et al., 1993; Pihlajamaki et al., 2004; Barense et al., 2005, 2007, 2010; Lee et al., 2005a,c, 2006a,b; Taylor et al., 2007; Lee et al., 2008). This work has motivated representational accounts of memory in which structures in the MTL are responsible for forming, storing and remembering object and scene representations, particularly when these exemplars have overlapping visual feature conjunctions (Emergent Memory Account, Graham et al., 2010; Representational-Hierarchical View, Saksida and Bussey, 2010). These accounts uniquely predict that $\operatorname{PrC}$ can support memory for object-context associations in the way demonstrated here. Although the specific computational mechanisms for how a single representation can support different memory processes remains unclear, this issue has been addressed in two recent papers (Berry et al., 2008; Greve et al., 2010).

Considering the main premise of representational accountsthat the PrC and $\mathrm{HC}$ care differentially about object and scene processing, particularly when the featural similarity of objects is high (Bussey and Saksida, 2002; Bussey et al., 2003; Lee et al., 2005c; Barense et al., 2005, 2007, 2010; Devlin and Price, 2007; Mundy et al., 2009)—can help explain differences between our findings and other related fMRI experiments. First, we took care to employ objects with visual and semantic similarities across the two encoding tasks, thereby placing an increased demand on the type of visual processing dependent upon PrC. Second, we avoided any encoding task that stressed spatial processing, and that might require the HC (O'Keefe, 1976; O'Keefe and Nadel, 1978; Winters et al., 2004; Lee et al., 2005b). In two studies where the $\mathrm{HC}$ was found to be involved in memory during encoding of context for novel object stimuli (Cansino et al., 2002; Uncapher and Rugg, 2009), participants encoded object location changes across trials. There is evidence that moving objects on a screen elicits HC activity (Pihlajamaki et al., 2004; Kumaran and Maguire, 2007), and this raises the possibility that the lack of context memory effects in $\mathrm{PrC}$ in these experiments could reflect the use of a spatial encoding task and/or that the objects used did not contain a sufficient level of visual/semantic similarity.

Although we found no $\mathrm{HC}$ involvement in memory in our fROI analysis, whole-brain comparisons revealed subthreshold $\mathrm{HC}$ activation that differentiated between correct and incorrect context judgments, mirroring the stronger $\mathrm{HC}$ findings reported previously, across different stimulus types (Cansino et al., 2002; Davachi et al., 2003; Ranganath et al., 2004; Staresina and Davachi, 2006, 2008; Uncapher and Rugg, 2009; Staresina et al., 2011). These outcomes have been taken to support the view that the anterior HC performs a domain-general role in supporting memory for context. In this study, however, the differences in activation evident across correct versus incorrect context judgments were no longer reliable when we controlled for item-memory strength (see also Kirwan et al., 2008; although Cohn et al., 2009 and Montaldi et al., 2006 found HC strength matched context effects for scenes and words, respectively, albeit at retrieval). More research is clearly required to assess the specific role of the $\mathrm{HC}$ in memory for context, and memory for different kinds of complex visual stimuli.

In summary, we have provided novel evidence of $\mathrm{PrC}$ contributions to memory for context when item strength is held constant. These findings are difficult to reconcile with variants of dual-process views of MTL function (Diana et al., 2007; Mayes et al., 2007; Ranganath, 2010), but are consistent with predictions from representational accounts (Graham et al., 2010; Saksida and Bussey, 2010), in which the $\operatorname{PrC}$ processes and stores representa- 
tions of objects that can support both perceptual and mnemonic operations.

\section{References}

Aggleton JP, Brown MW (1999) Episodic memory, amnesia and the hippocampal-anterior thalamic axis. Behav Brain Sci 22:425-489.

Awipi T, Davachi L (2008) Content-specific source encoding in the human medial temporal lobe. J Exp Psychol Learn Mem Cogn 34:769-779.

Barense MD, Bussey TJ, Lee AC, Rogers TT, Davies RR, Saksida LM, Murray EA, Graham KS (2005) Functional specialization in the human medial temporal lobe. J Neurosci 25:10239-10246.

Barense MD, Gaffan D, Graham KS (2007) The human medial temporal lobe process online representations of complex objects. Neuropsychologia 45:2963-2974.

Barense MD, Henson RN, Lee AC, Graham KS (2010) Medial temporal lobe activity during complex discriminations of faces, objects and scenes: effects of viewpoint. Hippocampus 20:389-401.

Beckmann CF, Jenkinson M, Smith SM (2003) General multilevel linear modeling for group analysis in FMRI. Neuroimage 20:1052-1063.

Berry CJ, Shanks DR, Henson RN (2008) A unitary signal-detection model of implicit and explicit memory. Trends Cogn Sci 12:367-373.

Brown MW, Aggleton JP (2001) Recognition memory: what are the roles of the perirhinal cortex and hippocampus? Nat Rev Neurosci 2:51-61.

Brown MW, Warburton EC, Aggleton JP (2010) Recognition memory: material, processes and substrates. Hippocampus 20:1228-1244.

Bussey TJ, Saksida LM (2002) The organization of visual object representations: a connectionist model of effects of lesions in perirhinal cortex. Eur J Neurosci 15:355-364.

Bussey TJ, Saksida LM, Murray EA (2003) Impairments in visual discrimination after perirhinal cortex lesions: testing 'declarative' vs. 'perceptual-mnemonic' views of perirhinal cortex function. Eur J Neurosci 17:649-660.

Cansino S, Maquet P, Dolan RJ, Rugg MD (2002) Brain activity underlying encoding and retrieval of source memory. Cereb Cortex 12:1048-1056.

Cohn M, Moscovitch M, Lahat A, McAndrews MP (2009) Recollection versus strength as the primary determinant of hippocampal engagement at retrieval. Proc Natl Acad Sci U S A 106:22451-22455.

Cowell RA, Bussey TJ, Saksida LM (2010) Functional dissociations within the ventral object-processing pathway: Cognitive modules or a hierarchical continuum? J Cogn Neurosci 22:2460-2479.

Daselaar SM, Fleck MS, Cabeza R (2006) Triple dissociation in the medial temporal lobes: Recollection, familiarity and novelty. J Neurophysiol 96:1902-1911.

Davachi L (2006) Item, context and relational episodic encoding in humans. Curr Opin Neurobiol 16:693-700.

Davachi L, Mitchell JP, Wagner AD (2003) Multiple routes to memory: distinct medial temporal lobe processes build item and source memories. Proc Natl Acad Sci U S A 100:2157-2162.

Devlin JT, Price CJ (2007) Perirhinal contributions to human visual perception. Curr Biol 17:1484-1488.

Diana RA, Yonelinas AP, Ranganath C (2007) Imaging recollection and familiarity in the medial temporal lobe: a three-component model. Trends Cogn Sci 11:379-386.

Diana RA, Yonelinas AP, Ranganath C (2010) Medial temporal lobe activity during source retrieval reflects information type, not memory strength. J Cogn Neurosci 22:1808-1818.

Downing PE, Chan AW, Peelen MV, Dodds CM, Kanwisher N (2006) Domain specificity in visual cortex. Cereb Cortex 16:1453-1461.

Duarte A, Henson RN, Graham KS (2011) Stimulus content and the neural correlates of source memory. Brain Res 1373:110-123.

Epstein RA, Higgins JS, Parker W, Aguirre GK, Cooperman S (2006) Cortical correlates of face and scene inversion: a comparison. Neuropsychologia 44:1145-1158.

Giovanello KS, Keane MM, Verfaellie M (2006) The contribution of familiarity to associative memory in amnesia. Neuropsychologia 44:1859-1865.

Graham KS, Barense MD, Lee AC (2010) Going beyond LTM in the MTL: a synthesis of neuropsychological and neuroimaging findings on the role of the medial temporal lobe in memory and perception. Neuropsychologia 48:831-853.

Greenhouse GW, and Geisser S (1959) On methods in the analysis of profile data. Psychometrika 24:95-112.
Greve A, Donaldson DI, van Rossum MC (2010) A single-trace dualprocess model of episodic memory: a novel computational account of familiarity and recollection. Hippocampus 20:235-251.

Haskins AL, Yonelinas AP, Quamme JR, Ranganath C (2008) Perirhinal cortex supports encoding and familiarity-based recognition of novel associations. Neuron 59:554-560.

Insausti R, Juottonen $\mathrm{K}$, Soininen $\mathrm{H}$, Insausti AM, Partanen $\mathrm{K}$, Vainio $\mathrm{P}$, Laakso MP, Pitkänen A (1998) MR volumetric analysis of the human entorhinal, perirhinal, and temporopolar cortices. AJNR Am J Neuroradiol 19:659-671.

Jenkinson M (2003) Fast, automated, N-dimensional phase-unwrapping algorithm. Magn Reson Med 49:193-197.

Jenkinson M, Smith S (2001) A global optimisation method for robust affine registration of brain images. Med Image Anal 5:143-156.

Jenkinson M, Bannister P, Brady M, Smith S (2002) Improved optimization for the robust and accurate linear registration and motion correction of brain images. Neuroimage 17:825-841.

Jezzard P, Balaban RS (1995) Correction for geometric distortion in echo planar images from B0 field variations. Magn Reson Med 34:65-73.

Kensinger EA, Schacter DL (2006) Amygdala activity is associated with the successful encoding of item, but not source, information for positive and negative stimuli. J Neurosci 26:2564-2570.

Kirwan CB, Wixted JT, Squire LR (2008) Activity in the medial temporal lobe predicts memory strength, whereas activity in the prefrontal cortex predicts recollection. J Neurosci 28:10541-10548.

Kirwan CB, Wixted JT, Squire LR (2010) A demonstration that the hippocampus supports recollection and familiarity. Proc Natl Acad Sci U S A 107:344-348.

Kumaran D, Maguire EA (2007) Match mismatch processes underlie human hippocampal responses to associative novelty. J Neurosci 27:8517-8524.

Lee AC, Barense MD, Graham KS (2005a) The contribution of the human medial temporal lobe to perception: bridging the gap between animal and human studies. Q J Exp Psychol 58B:300-325.

Lee AC, Buckley MJ, Pegman SJ, Spiers H, Scahill VL, Gaffan D, Bussey TJ, Davies RR, Kapur N, Hodges JR, Graham KS (2005b) Specialization in the medial temporal lobe for processing of objects and scenes. Hippocampus 15:782-797.

Lee AC, Bussey TJ, Murray EA, Saksida LM, Epstein RA, Kapur N, Hodges JR, Graham KS (2005c) Perceptual deficits in amnesia: challenging the medial temporal lobe 'mnemonic' view. Neuropsychologia 43:1-11.

Lee AC, Bandelow S, Schwarzbauer C, Henson RN, Graham KS (2006a) Perirhinal cortex activity during visual object discrimination: an eventrelated fMRI study. Neuroimage 33:362-373.

Lee AC, Buckley MJ, Gaffan D, Emery T, Hodges JR, Graham KS (2006b) Differentiating the roles of the hippocampus and perirhinal cortex in processes beyond long-term declarative memory: a double dissociation in dementia. J Neurosci 26:5198-5203.

Lee AC, Scahill VL, Graham KS (2008) Activating the medial temporal lobe during oddity judgment for faces and scenes. Cereb Cortex 18:683-696.

Mayes A, Montaldi D, Migo E (2007) Associative memory and the medial temporal lobes. Trends Cogn Sci 11:126-135.

Meunier M, Bachevalier J, Mishkin M, Murray EA (1993) Effects on visual recognition of combined and separate ablations of the entorhinal and perirhinal cortex in rhesus monkeys. J Neurosci 13:5418-5432.

Montaldi D, Mayes AR (2010) The role of recollection and familiarity in the functional differentiation of the medial temporal lobes. Hippocampus 20:1291-1314.

Montaldi D, Spencer TJ, Roberts N, Mayes AR (2006) The neural system that mediates familiarity memory. Hippocampus 16:504-520.

Mundy ME, Honey RC, Downing PE, Wise RG, Graham KS, Dwyer DM (2009) Material-independent and material-specific activation in functional MRI after perceptual learning. NeuroReport 20:1397-1401.

O'Keefe J (1976) Place units in the hippocampus of the freely moving rat. Exp Neurol 51:78-109.

O'Keefe J, Nadel L (1978) The hippocampus as a cognitive map. Oxford: Oxford UP.

O’Neil EB, Cate AD, Köhler S (2009) Perirhinal cortex contributes to accuracy in recognition memory and perceptual discriminations. J Neurosci 29:8329-8334.

Park H, Rugg MD (2011) Neural correlates of encoding within- and acrossdomain interitem associations. J Cogn Neurosci 23:2533-2543. 
Peelen MV, Downing PE (2005) Within-subject reproducibility of category specific activation with functional MRI. Hum Brain Mapp 25:402-408.

Pihlajamäki M, Tanila H, Könönen M, Hänninen T, Hämäläinen A, Soininen $\mathrm{H}$, Aronen HJ (2004) Visual presentation of novel objects and new spatial arrangements of objects differentially activates the medial temporal lobe subareas in humans. Eur J Neurosci 19:1939-1949.

Quamme JR, Yonelinas AP, Norman KA (2007) Effect of unitization on associative recognition in amnesia. Hippocampus 17:192-200.

Ranganath C (2010) A unified framework for the functional organization of the medial temporal lobes and the phenomenology of episodic memory. Hippocampus 20:1263-1290.

Ranganath C, Yonelinas AP, Cohen MX, Dy CJ, Tom SM, D'Esposito M (2004) Dissociable correlates of recollection and familiarity within the medial temporal lobes. Neuropsychologia 42:2-13.

Saksida LM, Bussey TJ (2010) The representational-hierarchical view of amnesia: translation from animal to human. Neuropsychologia 48:2370-2384.

Saksida LM, Bussey TJ, Buckmaster CA, Murray EA (2006) No effect of hippocampal lesions on perirhinal cortex-dependent feature-ambiguous visual discriminations. Hippocampus 16:421-430.

Smith SM (2002) Fast and automated brain extraction. Hum Brain Mapp $17: 143-155$

Staresina BP, Davachi L (2006) Differential encoding mechanisms for subsequent associative recognition and free recall. J Neurosci 26:9162-9172.

Staresina BP, Davachi L (2008) Selective and shared contributions of the hippocampus and perirhinal cortex to episodic item and associative encoding. J Cogn Neurosci 20:1478-1489.

Staresina BP, Davachi L (2010) Object unitization and associative memory formation are supported by distinct brain regions. J Neurosci 30:9890-9897.

Staresina BP, Duncan KD, Davachi L (2011) Perirhinal and parahippocampal cortices differentially contribute to later recollection of object- and scene-related event details. J Neurosci 31:8739-8747.
Taylor KJ, Henson RN, Graham KS (2007) Recognition memory for faces and scenes in amnesia: dissociable roles for medial temporal lobe structures. Neuropsychologia 45:2428-2438.

Uncapher MR, Rugg MD (2009) Selecting for memory? The influence of selective attention on the mnemonic binding of contextual Information. J Neurosci 29:8270-8279.

Watson C, Andermann F, Gloor P, Jones-Gotman M, Peters T, Evans A, Olivier A, Melanson D, Leroux G (1992) Anatomic basis of amygdaloid and hippocampal volume measurement by magnetic resonance imaging. Neurology 42:1743-1750.

Winters BD, Forwood SE, Cowell RA, Saksida LM, Bussey TJ (2004) Double dissociation between the effects of peri-postrhinal cortex and hippocampal lesions on tests of object recognition and spatial memory: heterogeneity of function. J Neurosci 24:5901-5908.

Wixted JT (2007) Dual-process theory and signal-detection theory of recognition memory. Psychol Rev 114:152-176.

Wixted JT, Mickes L, Squire LR (2010) Measuring recollection and familiarity in the medial temporal lobe. Hippocampus 20:1195-1205.

Woolrich MW, Ripley BD, Brady M, Smith SM (2001) Temporal autocorrelation in univariate linear modeling of fMRI data. Neuroimage 14:1370-1386.

Woolrich MW, Behrens TE, Beckmann CF, Jenkinson M, Smith SM (2004) Multilevel linear modeling for FMRI group analysis using Bayesian inference. Neuroimage 21:1732-1747.

Yonelinas AP (1999) The contribution of recollection and familiarity to recognition and source-memory judgments: a formal dual-process model and an analysis of receiver operating characteristics. J Exp Psychol Learn Mem Cogn 25:1415-1434.

Yonelinas AP, Otten LJ, Shaw KN, Rugg MD (2005) Separating the brain regions involved in recollection and familiarity in recognition memory. J Neurosci 25:3002-3008. 